\title{
Resistência de união dos sistemas adesivos em dentina úmida e em dentina seca: revisão integrativa
}

\author{
Union endurance of adhesive systems in humid dentin and dry dentin: integrative review \\ Resitencia de la unión de los sistemas adhesivos en dentina húmeda y en dentina seca: revisión
}

integradora

Recebido: 14/03/2021 | Revisado: 21/03/2021 | Aceito: 24/03/2021 | Publicado: 01/04/2021

Hiully Karydja Câmara Oliveira

ORCID: https://orcid.org/0000-0001-6845-4592

Universidade do Estado do Rio Grande do Norte, Brasil

E-mail: hiully_karydja1995@hotmail.com

Isabela Pinheiro Cavalcanti Lima

ORCID: https://orcid.org/0000-0002-7681-9675

Universidade do Estado do Rio Grande do Norte, Brasi

E-mail: belapcl@yahoo.com.br

Hugo Matheus Câmara Oliveira

ORCID: https://orcid.org/0000-0002-2672-685X

Universidade do Estado do Rio Grande do Norte, Brasil

E-mail: hugomathodonto@gmail.com

Nara Grazieli Martins Lima

ORCID: https://orcid.org/0000-0002-4436-8785

Universidade do Estado do Rio Grande do Norte, Brasil

E-mail: naragrazieli@gmail.com

Talita da Silva Pinto

ORCID: https://orcid.org/0000-0002-2911-3549 Universidade do Estado do Rio Grande do Norte, Brasil

E-mail: t_alitapinto@outlook.com

Matheus da Silva Regis

ORCID: https://orcid.org/0000-0001-9402-6030 Universidade do Estado do Rio Grande do Norte, Brasil

E-mail: matheussregis@gmail.com

Heloísa Pereira de Medeiros

ORCID: https://orcid.org/0000-0001-5769-4988

Universidade do Estado do Rio Grande do Norte, Brasil

E-mail: heloisap.medeiros@gmail.com

\begin{abstract}
Resumo
A adesão à dentina é dificultada por se tratar de uma estrutura com alta composição hídrica, ao longo dos anos os protocolos clínicos, assim como os sistemas adesivos foram sendo alterados, visando melhorar a resistência de união e simplificar a técnica. Por meio de revisão integrativa, discorre-se aqui sobre a resistência de união dos sistemas adesivos em dentina úmida e em dentina seca visando sugerir melhorias dos protocolos clínicos. Selecionou-se os artigos através da biblioteca virtual em saúde (BVS), obtendo-os das bases MEDLINE, LILACS, Biblioteca Brasileira de Odontologia (BBO), além de buscas no Google ${ }^{\circledR}$ Scholar, usando os termos indexados: "Dentin", "Dentina", "Dentin-Bonding Agents" e "Adesivos dentinários". Incluiu-se artigos publicados entre 2013 e 2020. Os estudos selecionados poderiam ser clínicos ou laboratoriais, em dentina úmida e em dentina seca, usando testes de resistência à tração, microtração ou cisalhamento nos estudos in vitro, com os sistemas adesivos convencionais, autocondicionantes e/ou universais. Foram excluídos artigos curtos, pôsteres ou revisões de literatura. Encontrou-se 969 artigos, sendo selecionados 8 deles, segundo os critérios de inclusão, todos os estudos abordavam o grau de umidade da dentina como um fator limitante na qualidade de adesão, assim como o conhecimento do operador a respeito da composição do sistema adesivo utilizado. Os sistemas adesivos universais foram eficazes tanto em dentina úmida quanto em dentina seca, já os sistemas adesivos convencionais foram eficientes apenas em dentina úmida.
\end{abstract}

Palavras-chave: Dentina; Protocolos clínicos; Resistência de união; Sistemas adesivos.

\section{Abstract}

Adhesion to dentin is difficult because it is a structure with high water composition, over the years the clinical protocols, as well as the adhesive systems have been changed, in order to improve the union endurance and simplify the technique. Through a integrative review, discuss the union endurance of adhesive systems in wet and dry dentin in order to improve clinical protocols. The selection of articles was carried out through the virtual health library (VHL), 
obtaining articles from the MEDLINE, LILACS, Biblioteca Brasileira de Odontologia (BBO) databases, in addition to searches on Google ${ }^{\circledR}$ Scholar, using the indexed terms: "Dentin", "Dentina", "Dentin-Bonding Agents" and "Adesivos dentinários". Articles published between 2013 and 2020 were included. The selected studies could be clinical or laboratory, in wet and dry dentin, using tensile, microtensile or shear strength tests in the studies in vitro, with conventional, self-etching and / or universal adhesive systems, published as short articles, posters or literature reviews were excluded. 969 were found, 8 articles were selected according to the inclusion criteria, all studies addressed the degree dentin moisture content as a limiting factor in the quality of adhesion, as well as the operator's knowledge about the composition of the adhesive system used. Universal adhesive systems were effective on both wet and dry dentin, conventional adhesive systems were efficient only on wet dentin.

Keywords: Dentin; Clinical protocols; Union endurance; Adhesive systems.

\section{Resumen}

La adherencia a la dentina es obstaculizada porque es una estructura con alta composición hídrica, a lo largo de los años los protocolos clínicos, así como los sistemas adhesivos han sido cambiados, con el objetivo de mejorar la resistencia de la unión y simplificar la técnica. Por medio de una revisión integradora, exponer sobre la resistencia de la unión de los sistemas adhesivos en dentina húmeda y en dentina seca com el objetivo de mejorar los protocolos clínicos. La selección de los artículo fue realizada a través de a biblioteca virtual en salud (BVS), consiguiendo artículos de las bases MEDLINE, LILACS, Biblioteca Brasileira de Odontologia (BBO), además de búsquedas en Google ${ }^{\circledR}$ Scholar, utilizando los términos indexados: "Dentin", "Dentina", "Dentin-Bonding Agents" e "Adesivos dentinários". Han sido incluido artículos publicados entre 2013 y 2020. Los estudios seleccionados podrían ser clínicos o laboratoriales, en dentina húmeda y en dentina seca, utilizando testes de resistencia a la tracción, microtracción o cizallamiento en los estudios in vitro, com los sistemas adhesivos convencionales, autoacondicionantes y/o universales. Han sido excluídas publicaciones como artículos cortos, carteles o revisiones de literatura. Fueron encontrados 969, habiendo sido seleccionados 8 artículos según los criterios de inclusión, todos los estudios presentaron el grado de humedad de la dentina como un factor limitante en la calidad de adherencia, así como el conocimiento del operador respecto a de la composición del sistema adhesivo utilizado. Los sistemas adhesivos universales han sido efectivos tanto en dentina húmeda como en dentina seca, los sistemas adhesivos convencionales fueron eficientes sólo en dentina húmeda.

Palabras clave: Dentina; Protocolos clínicos; Resistencia de la unión; Sistemas adhesivos.

\section{Introdução}

$\mathrm{O}$ advento dos sistemas adesivos (SAs) revolucionou a Odontologia minimamente invasiva, viabilizando a conservação da estrutura dentária, eliminando a necessidade de preparos cavitários extensos (Buonocore, 1955; Nakabayashi et al, 1982). Esses materiais odontológicos apresentam um bom desempenho de união ao esmalte, porém, ainda demonstram algumas dificuldades de união à dentina (Carvalho et al, 2012).

Encontra-se no mercado vários tipos de sistemas adesivos, como os adesivos convencionais, os primeiros a serem utilizados, que necessitam de condicionamento ácido prévio, em seguida surgiram os autocondicionantes (self etch), e recentemente, surgiram os adesivos universais ou multimodais vendidos em frasco único, por serem multifuncionais, podem ser utilizados tanto pela técnica convencional quanto pela técnica autocondicionante como também podem ser usados com ou sem condicionamento do esmalte (Arinelli et al, 2016; Lopes et al, 2016). Os adesivos autocondicionantes excluem a necessidade de realizar o condicionamento ácido e a etapa de lavagem, reduzindo a sensibilidade técnica do procedimento (Giannini et al, 2015).

Nos SAs que realizam condicionamento ácido prévio, no esmalte a adesão ocorre por embricamento mecânico, devido à formação dos tags (microtags e macrotags) que são infiltrações do adesivo no tecido desmineralizado após o condicionamento ácido, mas os mecanismos de união à dentina são diferentes, principalmente devido a sua composição com abundância em água e material orgânico (Silva et al, 2010).

Alguns fatores podem influenciar na adesão: a técnica operatória, o tipo de adesivo, a umidade do meio e o substrato, que pode ser o esmalte ou a dentina (Isolan et al, 2014). Na dentina, mais especificamente, entre os fatores que podem alterar a qualidade de união está à penetração do sistema adesivo entre as fibras colágenas, bem também ao grau de conversão dos monômeros do adesivo (Nakabayashi et al, 1982; Pashley et al, 2000). Considerando essas adversidades, os pesquisadores 
estão sempre em busca de sistemas adesivos com técnica mais simples, diminuindo possíveis erros durante a aplicação (Avelar et al, 2019).

Em relação à umidade, sabe-se que o ressecamento dificulta a penetração do adesivo entre as fibras colágenas, já que as mesmas estão colapsadas (Kanca, 1992). Além disso, o excesso de umidade pode formar uma barreira que interfere a penetração (Nakajima et al, 2000). Dessa forma, esse estudo teve como objetivo avaliar a resistência de adesão na dentina úmida e na dentina seca de alguns sistemas adesivos disponíveis para uso clínico, através da análise de literaturas afins.

\section{Metodologia}

Trata-se de uma revisão integrativa, com pergunta formulada usando o acrônimo PICO, sendo que cada letra representa um componente da questão: P - população/problema; I - intervenção; C - comparador/controle; O desfecho/outcome (Quadro 1). Definida como: A respeito da resistência de união, o protocolo clínico de dentina úmida melhora a adesividade, se comparado ao protocolo de dentina seca?

Quadro 1. Descrição da estratégia PICO.

\begin{tabular}{|c|c|c|}
\hline ACRÔNIMO & DEFINIÇÃO & DESCRIÇÃO \\
\hline $\mathbf{P}$ & Problema & Dentina úmida \\
\hline $\mathbf{I}$ & Intervenção & Umidade da dentina \\
\hline $\mathbf{C}$ & Comparador & Dentina seca \\
\hline $\mathbf{O}$ & Desfecho & Resistência de união à dentina \\
\hline
\end{tabular}

Fonte: Autores (2020).

Esse estudo consistiu em busca ativa na Biblioteca Virtual em Saúde (BVS), obtendo artigos publicados na MEDLINE, LILACS e na Biblioteca Brasileira de Odontologia (BBO), utilizando os descritores em ciências da saúde (Decs), em inglês e português: "Dentin", "Dentina", "Dentin-Bonding Agents" e "Adesivos dentinários", estes foram combinados utilizando os operadores booleanos “AND” e “OR” (Quadro 2). Para aumentar a sensibilidade da pesquisa, usamos os filtros: Adesivos Dentinários, Dentina e Materiais Dentários. 
Quadro 2. Estratégias de busca utilizadas.

\begin{tabular}{|c|c|}
\hline BASE DE DADOS & DETALHES DA PESQUISA NA BVS \\
\hline MEDLINE & $\begin{array}{l}\text { ("Dentin-Bonding Agents" OR "adesivos dentinários") AND } \\
\text { (dentin OR dentina) AND ( fulltext:("1" OR "1" OR "1" OR } \\
\text { "1" OR "1" OR "1" OR "1" OR "1" OR "1" OR "1" OR "1" } \\
\text { OR "1") AND db:("MEDLINE") AND mj:("Adesivos } \\
\text { Dentinários" OR "Dentina" OR "Materiais Dentários") AND } \\
\text { la:("en" OR "pt" OR "es")) AND (year_cluster:[2013 TO } \\
\text { 2020]) }\end{array}$ \\
\hline LILACS & $\begin{array}{l}\text { ("Dentin-Bonding Agents" OR "adesivos dentinários") AND } \\
\text { (dentin OR dentina) AND ( fulltext:("1" OR "1" OR "1" OR } \\
\text { "1" OR "1" OR "1" OR "1" OR "1" OR "1" OR "1" OR "1" } \\
\text { OR "1" OR "1" OR "1") AND db:("LILACS") AND } \\
\text { mj:("Adesivos Dentinários" OR "Dentina" OR "Materiais } \\
\text { Dentários") AND la:("en" OR "pt" OR "es")) AND } \\
\text { (year_cluster:[2013 TO 2020]) }\end{array}$ \\
\hline BBO - Odontologia & $\begin{array}{l}\text { ("Dentin-Bonding Agents" OR "adesivos dentinários") AND } \\
\text { (dentin OR dentina) AND ( fulltext:("1" OR "1" OR "1" OR } \\
\text { "1" OR "1" OR "1" OR "1" OR "1" OR "1" OR "1" OR "1" } \\
\text { OR "1" OR "1" OR "1" OR "1" OR "1") AND db:("BBO") } \\
\text { AND mj:("Adesivos Dentinários" OR "Dentina" OR } \\
\text { "Materiais Dentários") AND la:("en" OR "pt" OR "es")) } \\
\text { AND (year_cluster:[2013 TO 2020]) }\end{array}$ \\
\hline
\end{tabular}

Fonte: Autores (2020).

Além disso, os artigos foram limitados compreendo os idiomas inglês, português e espanhol, e terem sido publicados entre 2013 e 2020. Outro ponto importante, é que eles deveriam estar disponíveis integral e gratuitamente. Para os artigos não encontrados na íntegra ou não obtidos pelos autores, ainda foi realizada uma pesquisa no Google® scholar na busca do texto completo. Também foram incluídos na pesquisa artigos encontrados nas referências dos artigos selecionados. Além disso, uma pesquisa mais ampla foi realizada no Google ${ }^{\circledR}$ Scholar visando abranger a literatura "cinzenta". Maior ênfase foi dada aos estudos realizados recentemente, para incluir os sistemas adesivos mais atuais. O período de busca foi de 23 de julho a 15 de agosto de 2020 .

Foram aceitos estudos de ensaios Clínicos controlados, relatos de casos, guia de prática clínica, estudos primários de pesquisa. Selecionaram-se os estudos que seguiam os seguintes critérios de inclusão:

1) Estudos com testes de resistência de união realizados em dentina úmida e seca;

2) Estudos que considerem dentição permanente e sadia;

3) Estudos que incluam sistemas adesivos convencionais, autocondicionantes e/ou universais ou mesmo uma comparação entre os protocolos clínicos;

4) Estudos cujo protocolo clínico ou laboratorial utilizou como pré-tratamento da dentina o ácido fosfórico, realizou a limpeza do substrato após o condicionamento com água;

5) Estudos cujo protocolo clínico ou laboratorial utilizou resina composta fotopolimerizável e técnica 
incremental;

6) Estudos que realizaram testes de resistência à tração, microtração ou cisalhamento;

Os estudos foram excluídos nos casos de:

7) Trabalhos publicados como artigo curtos, pôsteres ou revisões de literatura;

8) Estudos cujo protocolo clínico ou laboratorial utilizou novas técnicas de aplicação dos sistemas adesivos;

A primeira seleção dos artigos foi realizada a partir da leitura dos títulos, os artigos foram enviados para o gerenciador de referências (EndNot ${ }^{\circledR}$ Web), para verificação das duplicatas. A segunda seleção foi a partir da análise dos resumos e palavras-chave, por fim, a leitura dos textos completos. Para artigos com título e o resumo incompleto ou que não estavam claros, o texto na íntegra foi obtido para esclarecer qualquer dúvida. Os estudos selecionados foram analisados por dois revisores independentes, que verificaram quais artigos preenchiam os critérios de inclusão.

As principais informações sobre os estudos, como, autor principal, título do estudo, objetivos e principais resultados foram agrupados (Tabela 1). Além disso, os sistemas adesivos, os fabricantes e locação de fabricação também foram sintetizados (Tabela 2). A umidade da dentina, o sistema adesivo empregado e a resistência de união foram extraídas usando um formulário personalizado pelos autores do estudo (Tabela 3; Tabela 4). (fonte: TNR 10 - justificado - espaço 1,5).

\section{Resultados}

Após a busca nas bases de dados foram identificados 969 estudos (Figura 1). Após a leitura dos títulos e remoção dos artigos duplicados, restaram 36 artigos, após a leitura dos resumos, esse número foi reduzido para 16, sendo que 20 artigos foram excluídos, pois não se enquadravam nos critérios.

Após a leitura completa dos 16 artigos restantes, 8 artigos foram excluídos pelas seguintes razões: (1) estudos que não incluíram o grau de umidade da dentina (Barcellos et al, 2013; Chowdhury et al, 2019; Hass et al, 2013; Tsujimoto et al, 2017; Vinagre et al, 2020; Yamauchi et al, 2019) e (5) estudos que não realizaram os testes usando resina composta fotopolimerizável (Agee et al, 2015; Guan et al, 2016).

Nas buscas nas bases de dados de ciências da saúde e de acordo com os critérios de elegibilidade, 3 artigos foram selecionados (Leite et al, 2018; Perdigão et al, 2014; Mena-Serrano et al, 2013). No Google® Scholar, em busca da literatura "cinzenta", 5 artigos se encaixaram nos critérios de inclusão (Choi et al, 2017; Castillo et al, 2013; Dutra, 2018; Gomes, 2013; Leite, 2014), totalizando 8 artigos para esta revisão sistemática (Figura 1).

Todos os estudos abordaram a umidade da dentina e seguiam protocolos clínicos semelhantes. Entre os artigos incluídos 2 estudos são clínicos, randomizados e duplo-cego (Perdigão et al, 2014; Mena-Serrano et al, 2013), enquanto os outros foram realizados em in vitro (Leite et al, 2018; Choi et al, 2017; Castillo et al, 2013; Dutra, 2018; Gomes, 2013; Leite, 2014). 
Figura 1. Fluxograma da seleção dos estudos incluídos.
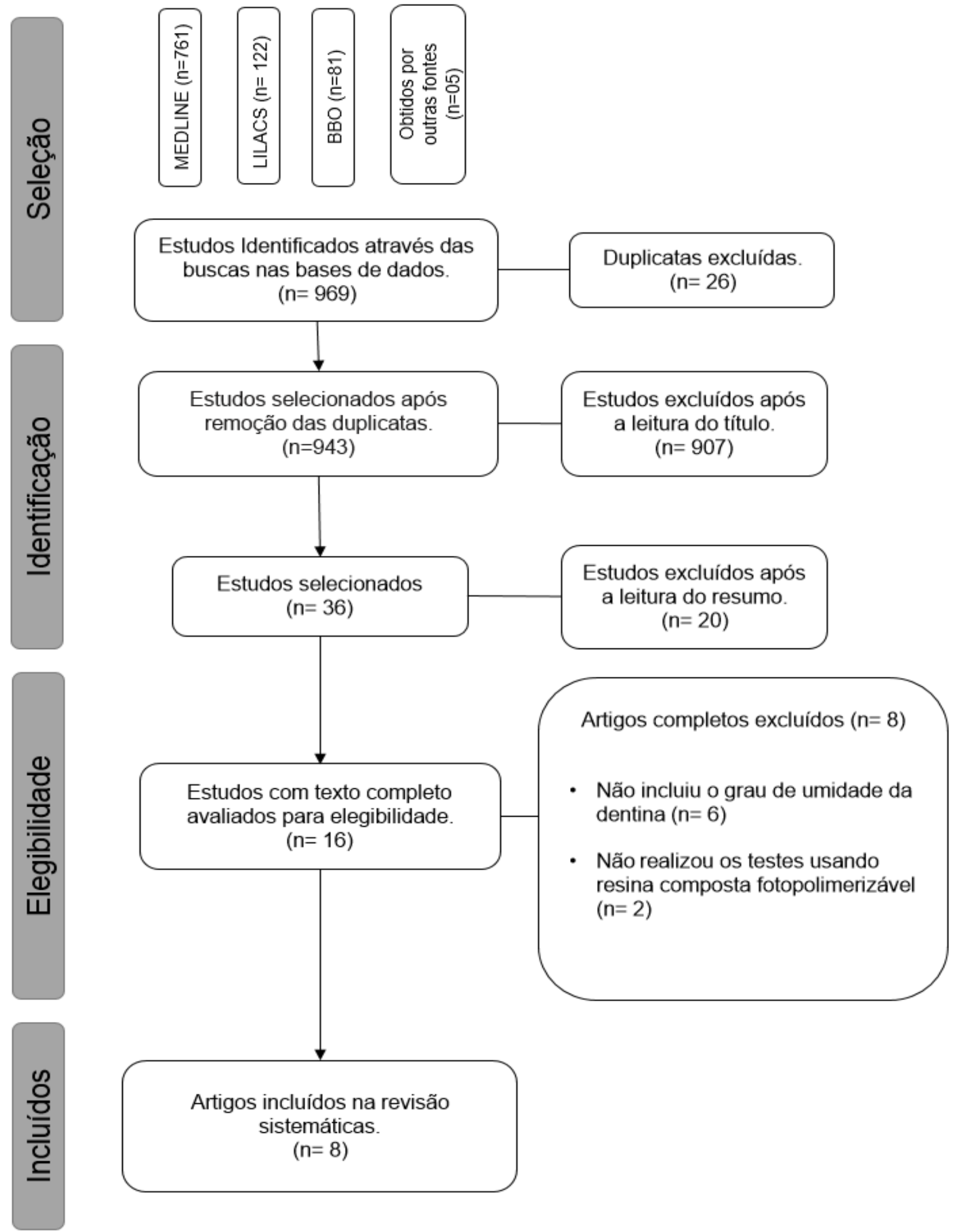

Fonte: Autores (2020). 
Tabela 1. Descrição dos principais dados dos artigos incluídos na revisão sistemática.

ID

Autor (es) /Ano

$\begin{array}{lll}\text { 1.Leite et al., } 2018 & \text { Laboratorial } & \begin{array}{l}\text { Avaliar a resistência de união à } \\ \text { microtração e citotoxidade } \\ \text { transdentinal usando diferentes } \\ \text { modos de hibridização. }\end{array} \\ & \text { Laboratorial } & \begin{array}{l}\text { Analisar a resistência de união } \\ \text { dos sistemas adesivos universais } \\ \text { à dentina utilizando diferentes } \\ \text { estratégias de adesão }\end{array}\end{array}$

$\begin{array}{lll}\text { 3.Choi el al., } 2017 & \text { Laboratorial } & \end{array}$

5.Gomes, $2013 \quad$ Laboratorial

6.Castillo et al., $2013 \quad$ Laboratorial

7.Perdigão et al., 2014

8.Mena-serrano et al., Clínico, 2013
Clínico, randomizado duplo-cego.

Avaliar a força de união de um sistema adesivo multimodo usando diferentes prétratamentos de dentina comparando com outros sistemas adesivos disponíveis no mercado.

Verificar o desempenho do sistema adesivo convencional em dentina seca e úmida, usando testes de tração.

Analisar o desempenho clínico

e de um sistema adesivo universal em lesões cervicais não cariosas, após 18 meses.

Analisar o desempenho clínico randomizado duplo-cego. e de um sistema adesivo universal em lesões cervicais não cariosas, após 6 meses.
A umidade da dentina não afetou a resistência de união nos SU, nos modos ER e SE. Contudo, a aplicação ER obteve melhor desempenho.

O sistema Single Bond universal obteve resultados positivos nas 3 estratégias de adesão, já o sistema adesivo Ambar Universal apresentou melhores resultados quando aplicado no modo ER em dentina úmida.

A umidade afeta a resistência de união adesiva à dentina dependendo do sistema adesivo universal empregado.

O sistema adesivo universal teve ótimo desempenho tanto em dentina úmida como em dentina seca, já o sistema adesivo convencional obteve bom desempenho apenas na dentina úmida.

$\mathrm{O}$ adesivo universal testado obteve um bom desempenho quanto usado tanto no modo SE como no ER em dentina úmida quanto em dentina seca.

A umidade interferiu positivamente da resistência à tração do sistema adesivo convencional.

O adesivo universal testado obteve bom desempenho clínico independente do protocolo adotado.

O adesivo universal testado obteve bom desempenho clínico independente do protocolo adotado.

LEGENDA: ID - identificação do artigo; ER - Protocolo de condicionamento e enxágue; SE - protocolo autocondicionante; SU Sistema adesivo Universal; 
Tabela 2. Composição, fabricantes e local de fabricação dos sistemas adesivos utilizados pelos autores.

\begin{tabular}{|c|c|c|}
\hline Sistema adesivo & Fabricante e local de fabricação & Composição \\
\hline Scotchbond Universal ${ }^{\circledR}$ & 3M ESPE, St Paul, MN, EUA & $\begin{array}{l}\text { Metacriloiloxidecil dihidrogênio fosfato (MDP), } \\
\text { dimetacrilato resinas, hidroxietilmetacrilato (HEMA), } \\
\text { modificado com metacrilato copolímero de ácido } \\
\text { polialquenóico(Copolímero Vitrebond TM), enchimento, } \\
\text { etanol, água, iniciadores, silano. }\end{array}$ \\
\hline Single Bond Universal® & 3M ESPE, Seefeld Alemanha & $\begin{array}{l}\text { 10-MDP, éster de ácido fosfórico monômero, HEMA, } \\
\text { silano, dimetacrilato, Vitrebond copolímero, enchimento, } \\
\text { etanol, água, iniciadores silano }\end{array}$ \\
\hline Ambar Universal ${ }^{\circledR}$ & $\begin{array}{l}\text { FGM, Joinville, Santa Catarina, } \\
\text { Brasil }\end{array}$ & $\begin{array}{l}\text { MDP (10-Metacriloiloxidecil dihidrogênio fosfato), } \\
\text { monômeros metacrílicos, fotoiniciadores, coiniciadores e } \\
\text { estabilizante, carga inerte (nanopartículas de sílica) e } \\
\text { veículo (etanol). }\end{array}$ \\
\hline G-Prêmio Bond $\AA$ & GC Corporation, Tóquio, Japão & $\begin{array}{l}\text { 10-MDP, éster de ácido fosfórico monômero, } \\
\text { dimetacrilato, 4-MET, MEPS, acetona, silício dióxido, } \\
\text { iniciadores }\end{array}$ \\
\hline All-Bond Universal ${ }^{\circledR}$ & Bisco Inc., Shaumburg, IL, EUA & $\begin{array}{l}\text { 10-MDP, monômero de éster de ácido fosfórico, Bis- } \\
\text { GMA, HEMA, etanol, água, iniciadores }\end{array}$ \\
\hline Prime \& Bond $2.1 \AA$ & Denstsply - Rio de Janeiro, Brasil & $\begin{array}{l}\text { UDMA, Bis-GMA, resinas di- e trimetacrilato, PENTA, } \\
\text { hidroxitolueno butilato, } 4 \text { etildi- metil amino benzoato, } \\
\text { fotoiniciadores, cetilamina hidro- fluorada e acetona. }\end{array}$ \\
\hline Adper Single Bond 2® & $3 M$ ESPE, St São Paulo, Brasil & $\begin{array}{l}\text { Resinas de dimetacrilato, hidroxietilmetacrilato(HEMA), } \\
\text { modificado com metacrilato copolímero de ácido } \\
\text { polialquenóico (Copolímero Vitrebond TM), enchimento, } \\
\text { etanol, água, iniciadores }\end{array}$ \\
\hline XP Bond ${ }^{\circledR}$ & $\begin{array}{l}\text { Dentsply® - Catanduva, São Paulo, } \\
\text { Brasil }\end{array}$ & $\begin{array}{l}\text { Resina TCB (Dimetacrilato Carboxílico modificado), } \\
\text { PENTA (Resina acrílica fosfatada modificada), Uretano } \\
\text { Dimetacrilato (UDMA), Trietilenoglicol, dimetacrilato } \\
\text { (TEGDMA), 2-hidroxietilmetacrilato (HEMA), } \\
\text { Estabilizadores, Etil-4- dimetilaminobenzoato, } \\
\text { Canforoquinona, Sílica Amorfa Funcionalizada e t- } \\
\text { butanol. }\end{array}$ \\
\hline
\end{tabular}

Fonte: Autores (2020).

Tabela 3. Descrição dos materiais empregados, umidade da dentina e resultados dos estudos laboratoriais.

\begin{tabular}{|c|c|c|c|c|c|c|c|c|}
\hline $\begin{array}{l}\text { I } \\
\text { D }\end{array}$ & ED & $\begin{array}{l}\text { Grupos } \\
(\mathbf{n}=)\end{array}$ & $\begin{array}{l}\text { Catego } \\
\text { ria do } \\
\text { SA }\end{array}$ & $\begin{array}{l}\text { Fabricante, Marca } \\
\text { e/ou nome do } \\
\text { sistema adesivo. }\end{array}$ & $\begin{array}{l}\text { Protocolo } \\
\text { de } \\
\text { aplicação }\end{array}$ & $\begin{array}{l}\text { Grau de } \\
\text { Umidade } \\
\text { da dentina }\end{array}$ & $\begin{array}{l}\text { Resistência de } \\
\text { União (Mpa) } \\
\text { Média } \pm \text { dp }\end{array}$ & Tipo de teste \\
\hline \multirow[t]{4}{*}{1} & \multirow{4}{*}{$\begin{array}{l}30 \\
\mathrm{ED}\end{array}$} & \multirow{4}{*}{$\mathrm{n}=5$} & \multirow[t]{4}{*}{ SU } & \multirow{4}{*}{$\begin{array}{l}\text { Scotchbond } \\
\text { Universal TM }\end{array}$} & \multirow[t]{2}{*}{ ER } & Úmida & $51,33 \pm 8,77$ & \multirow[t]{4}{*}{ Microtração } \\
\hline & & & & & & Seca & $51,97 \pm 9,37$ & \\
\hline & & & & & \multirow[t]{2}{*}{ SE } & Úmida & $9,7 \pm 0,97$ & \\
\hline & & & & & & Seca & $16,8 \pm 4,43$ & \\
\hline \multirow[t]{4}{*}{2} & \multirow{4}{*}{$\begin{array}{l}36 \\
\mathrm{ED}\end{array}$} & \multirow[t]{4}{*}{$\mathrm{n}=6$} & \multirow[t]{4}{*}{$\mathrm{SU}$} & \multirow{3}{*}{$\begin{array}{l}\text { Single } \\
\text { Universal }\end{array}$} & ER & Úmida & $48,05 \pm 10,27$ & \multirow[t]{4}{*}{ Microtração } \\
\hline & & & & & & Seca & $41,12 \pm 10,72$ & \\
\hline & & & & & SE & Úmida & $46,83 \pm 12,87$ & \\
\hline & & & & Ambar Universal & ER & Úmida & $44,46 \pm 12,36$ & \\
\hline
\end{tabular}




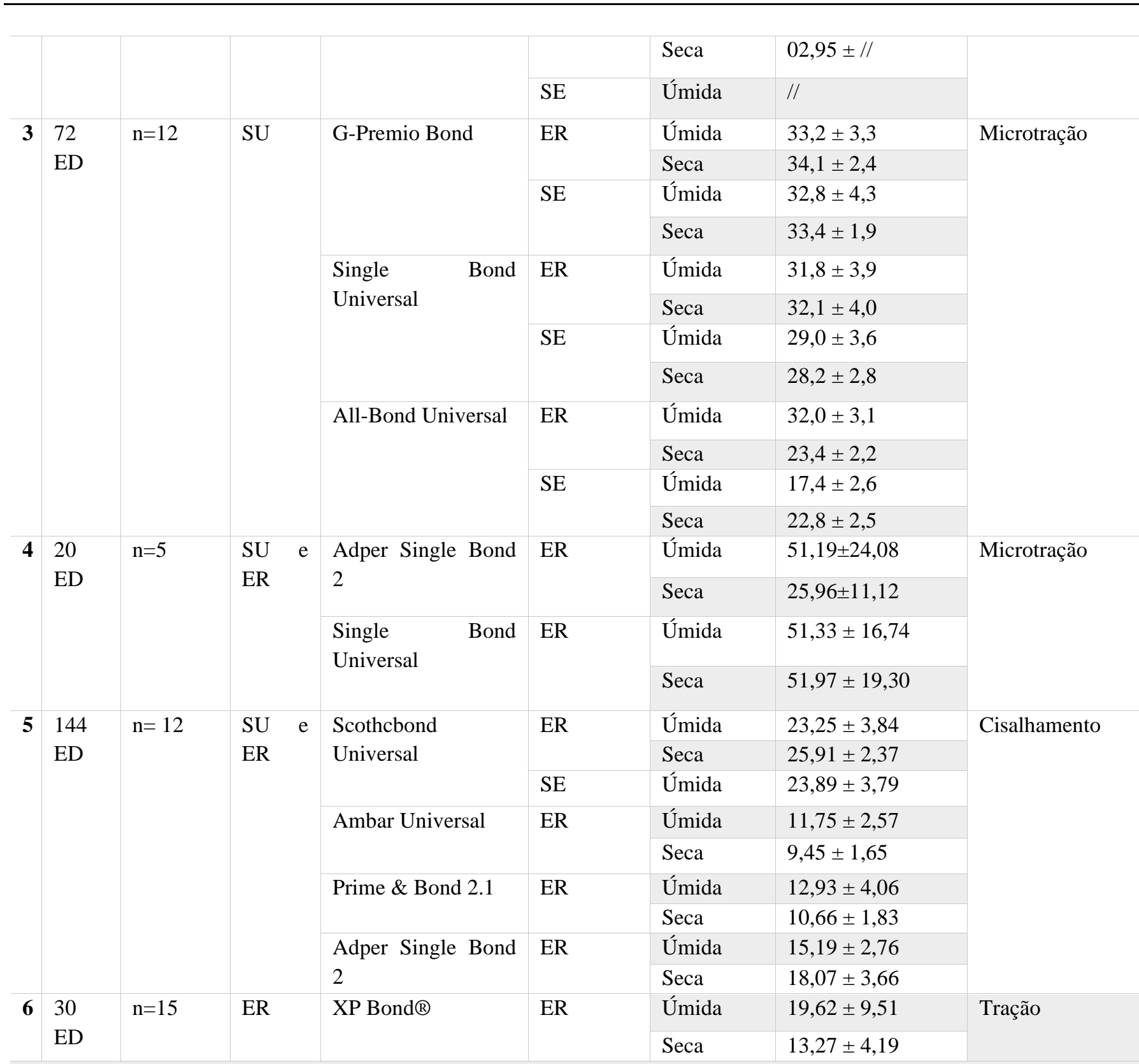

LEGENDA: ID - identificação do artigo; ED - Total de elementos dentários; SA - sistema adesivo; ER - Protocolo de condicionamento e enxágue; SE - protocolo autocondicionante; SU - Sistema adesivo Universal; // - sem dados; Média \pm dp - média e desvio padrão da resistência de união; Mpa = megapascal.

Observação: Em estudos realizados com vários tempos de secagem, consideramos o tempo 0s para dentina úmida e o último tempo de secagem para dentina seca.

Fonte: Autores (2020).

Tabela 4. Descrição dos materiais empregados, umidade da dentina e resultados dos estudos clínicos.

\begin{tabular}{|c|c|c|c|c|c|c|c|c|c|c|c|}
\hline ID & $\mathbf{P}$ & $\mathbf{R}$ & $\begin{array}{l}\text { Grupos } \\
(\mathbf{n}=)\end{array}$ & $\begin{array}{l}\text { Categoria } \\
\text { do SA }\end{array}$ & $\begin{array}{lr}\text { Fabricante, Marca } \\
\text { e/ou nome do } \\
\text { sistema adesivo. }\end{array}$ & $\begin{array}{l}\text { Protocolo } \\
\text { aplicação }\end{array}$ & de & $\begin{array}{ll}\text { Grau } & \text { de } \\
\text { Umidade } & \text { da } \\
\text { dentina } & \end{array}$ & $\mathbf{N r}$ & Np & PF \\
\hline \multirow[t]{4}{*}{7.} & \multirow[t]{4}{*}{$39 \mathrm{P}$} & \multirow[t]{4}{*}{$200 \mathrm{R}$} & \multirow[t]{4}{*}{$\mathrm{n}=50$} & \multirow[t]{4}{*}{ SU } & \multirow[t]{4}{*}{ Scotchbond Universal } & \multirow[t]{2}{*}{ ER } & & Úmida & 50 & 1 & 1 \\
\hline & & & & & & & & Seca & 50 & 0 & 1 \\
\hline & & & & & & SE $S$ es & & Seca & 50 & 1 & 1 \\
\hline & & & & & & SE & & Seca & 50 & 3 & 1 \\
\hline \multirow[t]{2}{*}{8.} & \multirow[t]{2}{*}{$39 \mathrm{P}$} & \multirow[t]{2}{*}{$200 \mathrm{R}$} & \multirow[t]{2}{*}{$\mathrm{n}=50$} & \multirow[t]{2}{*}{ SU } & \multirow[t]{2}{*}{ Scotchbond Universal } & \multirow{2}{*}{\multicolumn{2}{|c|}{ ER }} & Úmida & 50 & 1 & 0 \\
\hline & & & & & & & & Seca & 50 & 0 & 0 \\
\hline
\end{tabular}




\begin{tabular}{|l|l|c|c|c|}
\hline SE S es & $/ /$ & 50 & 0 & 0 \\
\hline ER & $/ /$ & 50 & 3 & 0 \\
\hline
\end{tabular}

LEGENDA: ID - identificação do artigo; P - amostra dos participantes; R - Amostra das restaurações; SA - sistema adesivo; ER Protocolo de condicionamento e enxágue; SE - Protocolo autocondicionante; SU - Sistema adesivo Universal; S es - Com condicionamento seletivo do esmalte; $\mathrm{Nr}$ - Número de restaurações realizadas; $\mathrm{Np}$ - Número de restaurações perdidas; PF - Pacientes faltantes no recal; // - Sem dados.

Fonte: Autores (2020).

\section{Discussão}

Esse estudo teve como propósito analisar estudos primários laboratoriais (Leite et al, 2018; Choi et al, 2017; Castillo et al, 2013; Dutra, 2018; Gomes, 2013; Leite, 2014) e estudos clínicos (Perdigão et al, 2014; Mena-Serrano et al, 2013), sobre o efeito da umidade na resistência de união de alguns sistemas adesivos, visando obter melhorias nos protocolos e assim obter boa adesão a dentina, buscando melhorar a longevidade das restaurações adesivas.

A simplificação das técnicas tem sido sugerida há anos, então o uso dos sistemas adesivos universais no modo autocondicionante é empregado para minimizar a sensibilidade operatória. Porém para Leite (2018) e Dutra (2018), os adesivos autocondicionantes mostraram desempenho laboratorial inferior aos sistemas adesivos convencionais de 3 passos, especialmente no quesito resistência de união. O estudo in vitro realizado por Gomes (2013), assim como os estudos clínicos relataram resultados satisfatórios no modo autocondicionante, porém é notório o maior número de restaurações perdidas nos estudos clínicos utilizando os sistemas adesivos autocondicionante em comparação ao sistema adesivo convencional de 3 passos (Perdigão et al, 2014; Mena-Serrano et al, 2013).

Atualmente, os sistemas adesivos universais têm ganhado espaço no mercado, os fabricantes pregam que eles são eficazes tanto na técnica convencional de 2 passos, em dentina úmida e em dentina seca, como na técnica autocondicionante, levando os pesquisadores a testarem a seu desempenho. Já existem disponíveis no mercado sistemas adesivos convencionais que prometem a adesão em dentina úmida e em dentina seca, como mostra o estudo de Castillo et al. (2013) que utilizou o sistema adesivo XP Bond, mas este adesivo mostrou-se eficaz apenas em dentina úmida, em desencontro com as recomendações do fabricante. De modo semelhante, Leite (2014) utilizou um sistema convencional Adper Single Bond 2 e obteve resultados melhores na dentina úmida.

Os sistemas adesivos universais testados em dentina úmida e em dentina seca obtiveram ótimo desempenho laboratorial (Leite et al, 2018; Gomes 2013; Leite, 2014). De acordo com Leite (2014) não há diferença estatística entre os sistemas adesivos universais usados por ele e os convencionais quando aplicado em dentina úmida, ainda relatou que os sistemas adesivos universais são tão eficazes quanto os convencionais, considerados padrão-ouro. Exceto os sistemas adesivos Ambar universal, All-Bond Universal, Prime \& Bond 2.1 que obtiveram resultados satisfatórios apenas em dentina úmida (Choi et al, 2013; Dutra, 2018; Gomes, 2013).

Como a dentina é naturalmente hidrofílica, a adesão micromecânica é dificultada, porém visando obter outros meios de adesão, os sistemas adesivos atuais incorporaram monômeros funcionais na composição, como o 10-MDP, o HEMA e atualmente adicionaram o copolímero Vitrobond. Tanto o 10-MDP como o Vitrobond se ligam ao cálcio presente na hidroxiapatita residual, com isso além da adesão micromecânica temos a adesão química, sendo assim, os resultados positivos obtidos com sistema adesivo Scothcbond Universal segundo o autor se deram pela presença do 10-MDP (Gomes, 2013). Comparando o sistema adesivo Single Bond Universal que contém 10-MDP na composição e o sistema convencional do grupo controle, o sistema adesivo universal se mostrou melhor, tanto em dentina úmida como em dentina seca (Leite, 2014).

Para Dutra (2018) o sistema adesivo Ambar Universal também contém o 10-MDP na composição e mesmo assim se mostrou ineficaz no modo autocondicionante e na técnica convencional em dentina seca, esse fato pode estar relacionado na 
quantidade de 10-MPD presente nesse sistema adesivo. Com isso, o autor concluiu que os componentes do sistema adesivo, podem interferir na resistência de união, seja pela quantidade de água, tipo de solvente e/ou presença do 10-MDP (Dutra, 2018).

O vitrobond é um copolímero que aumenta a estabilidade do adesivo mesmo em meio aquoso, mas para Choi (2017) o vitrobond não se mostrou um elemento primordial para melhorias na adesão. Dutra (2018) utilizou o sistema adesivo Single Bond Universal contendo o vitrobond na composição, e demonstrou que o mesmo foi eficaz na dentina úmida e seca. Corroborando com o estudo anteriormente citado, estudos clínicos relataram que o sistema adesivo Scotchbond Adesivo Universal obteve excelente resultados, com semelhanças aos adesivos "padrão ouro" avaliados, os autores ainda afirmaram que o sistema adesivo testado contém um baixo teor de 10-MDP sendo os resultados satisfatórios atribuídos ao vitrobond (Perdigão et al, 2014; Mena-Serrano et al, 2013). Sobre a HEMA, os autores dos estudos incluídos não abordaram a sua eficácia, ainda vale ressaltar que, apenas um componente do sistema adesivo não é fator determinante para melhorar a durabilidade de adesão.

Quanto ao solvente presente nos sistemas adesivos, podem ser utilizados o etanol, acetona ou água. Castillo et al. (2013) observaram resultados satisfatórios do sistema adesivo convencional cujo solvente era a água apenas em dentina úmida. Para tanto, Gomes (2018) relataram que o sistema adesivo prime\&Bond 2.1 constituído por acetona apresentou excelente resultado laboratoriais em dentina úmida. Isso é explicado pelo fato da acetona como solvente necessitar que a dentina tenha um grau de umidade ideal. Porém, o sistema adesivo G-premio Bond, que possui em sua composição a acetona, obteve ótimos resultados independente da secagem da dentina (Choi et al, 2017). Fato que leva a considerar que os solventes voláteis podem não ser tão sensíveis à presença ou à ausência de água. O XP Bond avaliado no estudo de Castillo et al. (2013), que tem como solvente o butanol terciário, mostrou-se eficaz apenas em dentina úmida, embora o fabricante enfatize que o solvente confere maior estabilidade e compatibilidade a esse sistema adesivo.

A resistência de união dos adesivos está relacionada a vários fatores, entre eles temos o tipo e a composição do próprio adesivo, assim como o substrato a que ele se liga. A presença de água na formulação dos sistemas adesivos é responsável pela ionização dos componentes ácidos e pela reexpansão da estrutura colágena, sendo um elemento essencial para a resistência de união, desse modo, os adesivos com menor teor de água apresentaram resultados inferiores, principalmente na secagem prolongada da dentina39, 43. Isso demonstra que o operador também precisa ter conhecimento sobre a formulação do sistema adesivo utilizado, mesmo usando sistemas adesivos com classificação semelhante.

Alguns autores (Gomes, 2013; Leite, 2014) ainda relacionaram a boa adesão dos sistemas adesivos universais à dentina úmida e à dentina seca com aplicação ativa, ou seja, a aplicação vigorosa no substrato.

A respeito dos testes de resistência de união, os estudos que utilizaram a microtração obtiveram resultados semelhantes utilizando o sistema adesivo universal no protocolo convencional de 2 passos, tanto em dentina úmida como em dentina seca; contudo, mostraram resultados inferiores para a técnica de autocondicionamento (Leite et al, 2018; Choi et al, 2017; Dutra, 2018; Leite, 2014). No teste de cisalhamento, foram satisfatórios em todos os modos de aplicação do adesivo, tanto o método convencional de 2 passos em dentina seca e em dentina úmida, e pelo método autocondicionante (Gomes, 2013). Já no teste de tração, os melhores resultados foram obtidos na dentina úmida, contudo, esse fato pode estar relacionado ao uso do sistema adesivo convencional (Castillo et al, 2013). Em suma, os resultados dos testes de resistência diferiram entre si.

\section{Conclusão}

A respeito da resistência de união, o protocolo clínico de dentina úmida melhora a adesividade, se comparado ao protocolo de dentina seca utilizando os sistemas adesivos universais. Os sistemas adesivos universais Scotchbond Universal, Single Bond Universal e o G-Prêmio Bond foram eficazes nos protocolos convencionais de 2 passos ou de condicionamento e 
enxágue em dentina úmida e em dentina seca, como também no modo autocondicionante.

Os sistemas adesivos que continham 10-MDP obtiveram ótimos resultados, já em relação ao vitrobond e a HEMA mais estudos precisam serem realizados.

A qualidade da adesão não depende de um único fator, mas da união de vários fatores, como a intensidade de aplicação do adesivo, o controle da etapa de evaporação do solvente, o controle da umidade de acordo com o sistema adesivo adotado e a formulação do adesivo.

O operador deve conhecer o sistema adesivo que está utilizando para determinar o protocolo ideal e controlar a umidade do substrato dentinário para aquele sistema adesivo. Por fim, mais estudos precisam serem realizados, principalmente a respeito dos componentes presentes nos sistemas adesivos.

\section{Referências}

Agee, K. A., Prakki, A., Abu-Haimed, T., Naguib, G. H., Nawareg, M. A., Tezvergil-Mutluay, A., Scheffel, D. L., Chen, C., Jang, S. S., Hwang, H., Brackett, M., Grégoire, G., Tay, F. R., Breschi, L., \& Pashley, D. H. (2015). Water distribution in dentin matrices: bound vs. unbound water. Dental materials: official publication of the Academy of Dental Materials, 31(3), 205-216.

Arinelli, A. M. D., Pereira, K. F., Prado, N. A. S. \& Rabello, T. B. (2016). Sistemas adesivos atuais. Rev. bras. odontol., 73(3): 242-46.

Avelar, W. V., Medeiros, A. F., Queiroz, A. M. de, Lima, D. A. S., Vasconcelos, M. G., \& Vasconcelos, R. G. (2019). Sistemas adesivos universais: alternativas de protocolos adesivos na união aos substratos dentários. Salusvita, 38(1): 133-153.

Barcellos, D. C., Batista, G. R., Silva, M. A., Pleffken, P. R., Rangel, P. M., Fernandes, V. V. J., Di Nicoló, R., \& Torres, C. R. (2013). Two-year clinical performance of self-etching adhesive systems in composite restorations of anterior teeth. Oper Dent., 38(3):258-66.

Buonocore, M. G. (1955). A simple method of increasing the adhesion of acrylic filling materials to enamel surfaces. Journal of dental research, 34(6), 849853.

Carvalho, R. C. C., Falcão, C. B., Conde, D. M., Marques, R.V. C., \& Ahid, F. J. M. (2012) Resistência de união de dois sistemas adesivos ao esmalte bovino. Odontol. Clín.-Cient., 11(1): 57-60.

Chowdhury, A. F. M. A., Saikaew, P., Matsumoto, M., Sano, H., \& Carvalho, R. M. (2019). Gradual dehydration affects the mechanical properties and bonding outcome of adhesives to dentin. Dent Mater J., 38(3):361-367.

Choi, A. N., Lee, J. H., Son, S. A., Jung, K. H., Kwon, Y. H., \& Park, J. K. (2017). Effect of Dentin Wetness on the Bond Strength of Universal Adhesives. Materials (Basel), 10(11):1224.

Castillo, K. A., Costa, S. R. M., Barros, R. M. G., Guerisoli, D. M. Z., \& Figueiredo, J. L. G. (2013). Resistência a Tração de um Sistema Adesivo em Dentina Seca e em Dentina úmida. Arch Health Invest., 2(4): 11-17.

Dutra, D. J. B. (2018). Avaliação da resistência de união de sistemas adesivos universais à dentina humana, utilizando diferentes estratégias de adesão. [Dissertação]. Universidade Federal de Minas Gerais: Faculdade de Odontologia.

Giannini, M., Makishi, P., Ayres, A. P. A., Vermelho, P. M., Fronza, B. M., Nikaido, T., \& Tagami, J. (2015). Self-Etch Adhesive Systems: A Literature Review. Braz. Dent. J., 26(1): 3-10.

Guan, R., Takagaki, T., Matsui, N., Sato, T., Burrow, M. F., Palamara, J., Nikaido, T., \& Tagami, J. (2016). Dentin bonding performance using Weibull statistics and evaluation of acid-base resistant zone formation of recently introduced adhesives. Dental materials journal, 35(4), 684-693.

Gomes, S. R. F. (2013). Resistência de união ao teste de cisalhamento de um sistema adesivo universal multi-modo. [Dissertação]. Universidade de Brasília, Brasília.

Hass, V., Dobrovolski, M., Zander-Grande, C., Martins, G. C., Gordillo, L. A., Rodrigues Accorinte, M., Gomes, O. M., Loguercio, A. D., \& Reis, A. (2013). Correlation between degree of conversion, resin-dentin bond strength and nanoleakage of simplified etch-and-rinse adhesives. Dental materials : official publication of the Academy of Dental Materials, 29(9), 921-928.

Isolan C. P., Valente, L. L., Münchow, E. A., Basso, G. R., Pimentel, A. H., Schwantz, J. K., da Silva, A. V., \& Moraes, R. R. (2014). Bond strength of a universal bonding agent and other contemporary dental adhesives applied on enamel, dentin, composite, and porcelain. Applied Adhesion Science, 2:(1):25.

Kanca, J., 3rd (1992). Improving bond strength through acid etching of dentin and bonding to wet dentin surfaces. Journal of the American Dental Association (1939), 123(9), 35-43.

Lopes, L. S., Malaquias, P., Calazans, F. S., Reis, A., Loguérico, A. D., \& Barceleiro, M. O. (2016). Protocolo das possibilidades técnicas de aplicação dos sistemas adesivos universais: Revisão de literatura com relato de caso. Rev. Bras. Odontol. 73(2): 173-7.

Leite, M. L. A. S., Costa, C. A. S., Duarte, R. M., Andrade, A. K. M., \& Soares, D. G. (2018). Bond Strength and Cytotoxicity of a Universal Adhesive According to the Hybridization Strategies to Dentin. Brazilian Dental Journal, 29(1), 68-75. 
Research, Society and Development, v. 10, n. 4, e10710413894, 2021

(CC BY 4.0) | ISSN 2525-3409 | DOI: http://dx.doi.org/10.33448/rsd-v10i4.13894

Leite, M. L. A. S. (2014). Avaliação de união à microtração de sistemas adesivos à dentina - Influência da umidade [Internet]. Universidade Federal da Paraíba - Faculdade de Odontologia, João Pessoa.

Mena-Serrano, A., Kose, C., De Paula, E. A., Tay, L. Y., Reis, A., Loguercio, A. D., \& Perdigão, J. (2013). A new universal simplified adhesive: 6-month clinical evaluation. J Esthet Restor Dent., 25(1):55-69.

Nakabayashi, N., Kojima, K., \& Masuhara, E. (1982). The promotion of adhesion by the infiltration of monomers into tooth substrates. Journal of biomedical materials research, 16(3), 265-273.

Nakajima, M., Kanemura, N., Pereira, P. N., Tagami, J., \& Pashley, D. H. (2000). Comparative microtensile bond strength and SEM analysis of bonding to wet and dry dentin. American journal of dentistry, 13(6), 324-328.

Pashley, D. H., Zhang, Y., Agee, K. A., Rouse, C. J., Carvalho, R. M., \& Russell, C. M. (2000). Permeability of demineralized dentin to HEMA. Dent Mater. 16:7-14.

Perdigão, J., Kose, C., Mena-Serrano, A. P., De Paula, E. A., Tay, L. Y., Reis, A., \& Loguercio, A. D. (2014). A new universal simplified adhesive: 18-month clinical evaluation. Oper Dent., 39(2):113-27.

Silva, E. O. S., Beltrani, F. C., Shibayana, R., Contreras, E. F. R., \& Hoeppner, E. F. R. (2010). Sistemas adesivos: conceito, aplicação e efetividade. Arq. Ciênc. Saúde UNIPAR, 14(1): 81-87.

Tsujimoto, A., Barkmeier, W. W., Takamizawa, T., Wilwerding, T. M., Latta, M. A., \& Miyazaki, M. (2017). Interfacial Characteristics and Bond Durability of Universal Adhesive to Various Substrates. Operative Dentistry. 42(2): E59-E70.

Vinagre, A., Ramos, J., Marques, F., Chambino, A., Messias, A., \& Mata, A. (2020). Randomized clinical trial of five adhesive systems in occlusal restorations: One-year results. Dent Mater J., 39(3):397-406.

Yamauchi, K., Tsujimoto, A., Jurado, C. A., Shimatani, Y., Nagura, Y., Takamizawa, T., Barkmeier, W. W., Latta, M. A., \& Miyazaki, M. (2019). Etch-andrinse vs self-etch mode for dentin bonding effectiveness of universal adhesives. Journal of oral science, 61(4), 549-553. 